\title{
Sources of Alaska Native Health Data and Statistics
}

\author{
Sigrid Brudie and Christy Garrett
}

Alaska Natives, as Alaska's indigenous people are known, live predominantly in rural communities with tiny populations, where small numbers make it difficult to collect meaningful health data. Information on the health status of Alaskans is mostly in the form of regional and state statistics. However, there are a few websites where data looking specifically at the health of Alaska Natives can be found. Those sites and their coverage are described here, starting with the most content-rich site regarding Alaska Natives.

\section{Alaska Native Epidemiology Center (EpiCenter), Alaska Native Tribal Health Consortium (ANTHC)}

http://www.anthctoday.org/epicenter/data/index.html

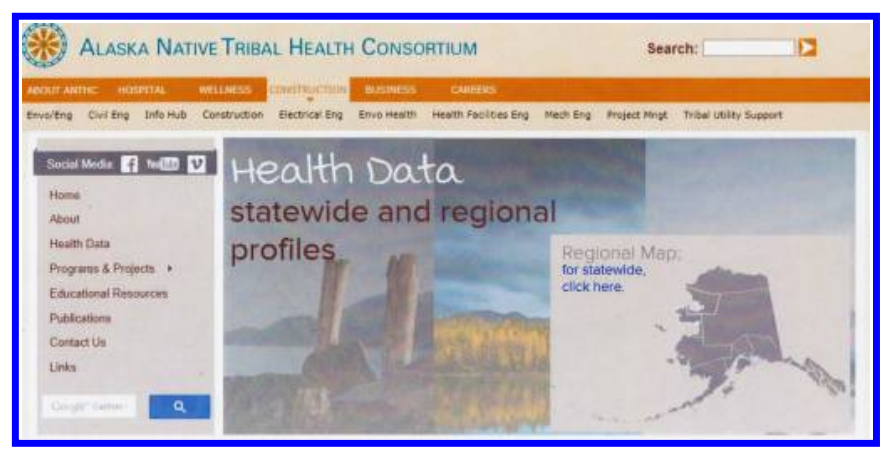

ANTHC's EpiCenter is 1 of 12 tribal epidemiology centers in the United States established by the Indian Health Service, the federal health program for American Indians and Alaska Natives. Data sharing is a core objective of the Center. The primary tool on the Health Data webpage is the Alaska Native Health Status Report, which provides data across a range of health indicators including adult and adolescent lifestyle risk factors, maternal and child health, cancer and cancer screening, immunizations, diabetes, infectious diseases, environmental health, and dental health. The core document is a 2009 print report that can be downloaded from the Center's website and provides a statewide overview. An interactive map of Alaska allows the user to click on any of the state's 12 Tribal Health Regions to access data for that region.

On each region's page, there are three tabs from which to choose. The page opens in the "current data" tab, which provides one-page fact sheets in the form of PDF files organized into eight categories: demographics, mortality, morbidity, maternal and child, adult health, adolescent health, prevention, and "other data."

Each fact sheet contains the most recent data available on a specific health issue. For example, clicking "Interior Region" brings up 78 PDF files, including one called "Unintentional Injury Deaths." The fact sheet starts with a definition, i.e., "total number of deaths due to unintentional injuries per 100,000 persons," followed by the corresponding goal from Healthy People 2020 (10-year national health objectives outlined by the US Department of Health and Human Services), in this case to "reduce unintentional injury death rate to 36.0 per 100,000 persons." Next is a bulleted list summarizing relevant data for Alaska Natives in the Interior Region, followed by a graph and a table representing the data over a 20 -year time period. Rates are compared for three groups-Alaska Natives in the region, Alaska Natives statewide, and US Whites - along with the Healthy People goal for that topic.

The "archived data" tab is currently empty for all regions but will eventually contain fact sheets and reports as they are replaced by newer ones.

The "full regional profile" tab provides a link to the most recent health status report for the region. Publication dates range from 2007 to 2012, with the AleutiansPribilofs Region report being the most recently updated. Average report length is 105 pages, and every report is full of data from a range of sources (Table 1).

ANTHC's EpiCenter is an essential starting point for anyone needing information on the health status of Alaska Natives. 
Table 1. Range of data found in regional profile reports.

\begin{tabular}{|c|c|}
\hline Source & Data accessed from: \\
\hline
\end{tabular}

\section{Alaska Center for Health Data and Statistics, State of Alaska Division of Public Health}

http://dhss.alaska.gov/dph/infocenter/Pages/default.aspx

The Alaska Center for Health Data and Statistics collects statewide information on births, deaths, newly diagnosed cases of cancer, injuries, occupational conditions and illnesses, and health behaviours. Information is categorized under 20 "topics of public health importance," from alcohol use to food safety. For example, clicking on the topic "obesity/nutrition/physical activity" brings up approximately 30 links to State of Alaska program status reports, studies, guides, fact sheets, and bulletins containing such data as "fruit and vegetable intake in Alaska" (2010 Alaska Department of Health and Social Services report).

State surveillance covers all Alaskans, so visitors to the site need to drill down to information specifically about Alaska Natives. A new tool on the website called "Informed Alaskans" makes it easier to find Alaska Native health data and statistics. It combines a database and query system with interactive maps for accessing data compiled by the Alaska Behavioral Risk Factor Surveillance System (BRFSS), a program put in place in 1991 as part of the Centers for Disease Control and Prevention's efforts to track health conditions and risk behaviors in the United States. "Informed Alaskans" allows users to break out Alaska BRFSS data by region-Public Health Region, Metro-Micropolitan Statistical Area, Borough ${ }^{2}$-Census Area, or Tribal Health Region - and further by health indicator and by race, so that health data for Alaska Natives can be isolated and compared.

\footnotetext{
${ }^{2}$ Unlike other states, Alaska uses boroughs rather than counties as geographic designations.
}

An additional link on the Center's website is to current and archived copies of the Epidemiology Bulletin, published since 1970 by the State of Alaska Section of Epidemiology. There are hundreds of bulletins indexed by category, many of which contain data on the health of Alaska Natives. For example, a bulletin from 2004 is entitled "Use of Traditional Foods in a Healthy Diet in Alaska: Risks in Perspective." This two-volume issue of the bulletin contains extensive charts and tables documenting methylmercury and PCB concentrations in traditional Alaska Native food sources. A bulletin from November 2013 called "Huffing in Alaska" provides data from the Alaska Youth Risk Behavior Survey. It compares adolescent inhalant abuse rates by type of school (traditional, alternative, corrective), race (Hispanic, White, Alaska Native), and Alaska Public Health Region (Interior, Gulf Coast, Anchorage-Mat-Su, Northern, Southwest, Southeast).

A significant amount of Alaska Native health data can be found on the Alaska Center for Health Data and Statistics website, and as the "Informed Alaskans" tool is perfected it will become easier to compare State of Alaska's Public Health Region data with ANTHC's Tribal Health Region data.

\section{The Center for Alaska Native Health Research (CANHR), University of Alaska, Fairbanks}

http://www.uaf.edu/canhr/

The Center for Alaska Native Health Research was established in 2001 through a five-year grant awarded by the National Institutes of Health to the University of Alaska, Fairbanks. The Center's research focuses on metabolic disorders, nutrition, obesity, cancer, and mental health in Alaska Natives. For example, colorectal cancer 
rates are high among Alaska Natives in northwest Alaska, and CANHR is intensifying its research into this disparity.

The Center conducts collaborative, community-based participatory research, encouraging tribal members to become coresearchers. CANHR has partnered with the Yukon-Kuskokwim Health Corporation (YKHC) on a number of projects. YKHC is a tribal organization that administers healthcare delivery to 58 rural communities in southwest Alaska. A recent CANHR-YKHC collaborative project dealt with suicide and substance abuse intervention efforts in Yup'ik communities in southwest Alaska. The goal was to explore how Alaska Native people use ancestral knowledge and cultural traditions to promote well-being in young people and their families.

The "Research Publications" page of the Center's website contains many citations to articles, books, theses, and dissertations that report results from research conducted at the Center.

CANHR seeks to share data gathered through its research projects. For confidentiality and intellectual property reasons, a data access application and sharing agreement are required. A list of current and past projects is available on CANHR's website as well as paperwork for requesting access to research data.

\section{Circumpolar Health Observatory (CircHOB)}

\section{http://circhob.circumpolarhealth.org/}

CircHOB is an international collaborative health information system that seeks to develop systematic, standardized, and consistent data collection and analysis for northern regions in all circumpolar countries, including health data on Alaska Natives. The Observatory is located at the Institute for Circumpolar Health Research in Yellowknife, NT. CircHOB has aggregated datasets from sources that include: Census and national population registries, vital statistics, morbidity and mortality datasets, and health surveys and other statistical reports. Subjects currently covered are population, fertility, mortality, reproductive outcomes, disease incidence, socioeconomic conditions, and health-related behaviors. The CircHOB website also offers interactive thematic mapping and graphing for selected datasets. Data are currently available for 2000-2009.

\section{Arctic Health}

http://arctichealth.nlm.nih.gov/

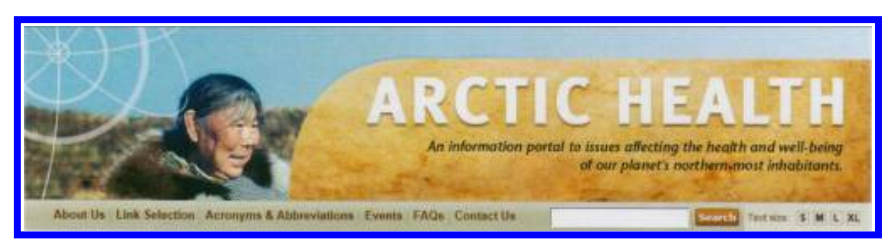

The Arctic Health website, managed jointly by the US National Library of Medicine and the Alaska Medical
Library at the University of Alaska Anchorage focuses on the health and well-being of Indigenous peoples of the circumpolar Arctic, including Alaska Natives. The site includes a growing section of links to health data sources. This complements other sections providing information about research conducted on climate change, food, air and water, and traditional healing. The site also has an extensive publications database, including grey literature that contains data from past health research projects. Other than the publications database, the site serves primarily as a portal, directing visitors through to other websites where the actual data can be found. In the "Research and Data" section, there are links to 18 research databases and portals that provide data as well as links to websites for 37 Arctic research groups where descriptions of current projects and contact information for principal investigators can be found. For example, on the "Research Organizations" page, one can find a link to the Institute for Circumpolar Health Studies website, where under "Research" then "Current Projects" there are descriptions of nine projects currently underway as well as researcher contact information. Current projects include "HIV/STI Evidence-Based Intervention for Alaska Native Communities," "Social and Physical Determinants of Circumpolar Health: A Meta-Analysis," and "Evaluating the Challenges to Self-Sufficiency Faced by TANF Clients in Alaska." The site also contains reports from previous research projects.

Because this portal pertains to Arctic health in general, not all links relate to the health of Alaska Natives. Finding data and statistics on the Arctic Health website therefore requires some browsing and sifting, but with the site's increasing focus on research and data it will likely become easier.

\section{Conclusion}

The researcher looking for Alaska Native health data and statistics is advised to start with the Alaska Native Epidemiology Center website as it is the most content-rich. For a comparison of Alaska Native health statistics with the general Alaska population, the new "Informed Alaskans" tool on the Alaska Center for Health Data and Statistics website is a helpful resource. The Center for Alaska Native Health Research does not provide open access to their research data but makes the data available through an application process. This might not be helpful for librarians or casual researchers looking for data, but there is a wealth of bibliographic information and project descriptions that can be accessed. Two websites that focus on health issues in the circumpolar north, Circumpolar Health Observatory and Arctic Health, are not specific to Alaska Natives, but the diligent searcher will find Alaska Native health data and statistics on those sites.

Research and data sources on Alaska Natives are becoming more numerous, and the hope is that access to the data will grow as well. 